\title{
Os benefícios da acupuntura no tratamento da ansiedade
}

Qualquer pessoa pode passar pelo transtorno de ansiedade, é um mal que atinge todas as classes sociais, ela se apresenta geralmente antes de eventos importantes, apresentações de negócios, quando estamos sendo analisados. Infelizmente quando não tratados esses sintomas podem se tornar uma anomalia negativa, como medos, e podem se tornar crônicos. Quando a vítima de ansiedade entra em estado crônico, elas podem ser atormentadas por ataques de pânico, pensamentos obsessivos, flashbacks de eventos traumáticos, pesadelos, ou vários sintomas físicos de medo. Existem tratamentos adequados e eficazes que podem sanar este problema, a acupuntura, medicina tradicional chinesa, que vem sendo utilizada como complemento para a medicina alopática, por meio de agulhas é uma aliada ao tratamento da ansiedade. Partindo dessa premissa o presente artigo de referência bibliográfica tem como objetivo atestar que a acupuntura auxilia no tratamento da ansiedade. Desta forma foi realizado pesquisas em artigos científicos e teses relacionados ao tema proposto.

Palavras-chave: Ansiedade; Medicina Tradicional Chinesa; Acupuntura.

\section{The benefits of acupuncture in anxiety treatment}

Anyone can go through anxiety disorder, it is an evil that reaches all social classes, it presents itself before important events, business presentations, when we are being analyzed. Unfortunately when untreated these symptoms can become a negative anomaly, such as fears, and can become chronic. When the anxiety victim goes into chronic condition, they may be plagued by panic attacks, obsessive thoughts, flashbacks of traumatic events, nightmares, or various physical symptoms of fear. There are adequate and effective treatments that can cure this problem, acupuncture, traditional Chinese medicine, which has been used as a supplement to allopathic medicine, through needles is an allied to the treatment of anxiety. Based on this premise, the present bibliographical article aims to attest that acupuncture helps in the treatment of anxiety. In this way, research was done on scientific articles and theses related to the proposed theme.

Keywords: Anxiety; Traditional Chinese Medicine; Acupuncture.

Topic: Fisioterapia

Reviewed anonymously in the process of blind peer.
Received: 25/06/2019

Approved: 15/09/2019

Leonardo Ricardo Franco

Instituto de Excelência de Educação e Saúde, Brasil

leonardofrancof@icloud.com

Danyelle Brito Cirqueira Queiroz

Instituto de Excelência de Educação e Saúde, Brasil

http://lattes.cnpq.br/2022337219722895

danycirqueira@hotmail.com

Referencing this:

FRANCO, L. R.; QUEIROZ, D. B. C.. Os benefícios da acupuntura no tratamento da ansiedade. Scire Salutis, v.9, n.3, p.8-15, 2019. DOI: http://doi.org/10.6008/CBPC2236-9600.2019.003.0002 


\section{INTRODUÇÃO}

O objetivo desta pesquisa é realizar por meio de levantamento bibliográfico uma revisão da literatura científica sobre os benefícios da acupuntura no tratamento da ansiedade. Desde os primórdios o homem convive com a ansiedade, ou seja, ele acordava já ansioso a procura da caça para sustentar a sua família, convivia também em cavernas abertas, com medo de que algum bicho viesse devorar seu ente querido ou de roubar a sua caça do dia. A ansiedade faz parte do homem, tanto do de antigamente quanto o atual.

$\mathrm{Na}$ atualidade o homem sofre de ansiedade, vivemos em um mundo competitivo e global, onde os problemas são imensos, falta de emprego, moradia, educação e saúde. É um novo distúrbio psíquico que a sociedade enfrenta. Uma sociedade voltada ao consumo e o acúmulo de riquezas, onde as relações interpessoais se tornam cada vez mais distantes, sendo que a pressão é um dos fatores na vida do homem. É um mundo de incertezas, e isto ocasiona a ansiedade, sendo esta prejudicial à saúde. Muitas pessoas recorrem a remédios, para sanar a ansiedade e acabam sendo consumidos por eles, sem uma prova de cura.

A medicina Tradicional Chinesa é uma ciência que trata as doenças do homem harmonicamente, especificamente a acupuntura, que se baseia no princípio de que o homem deve estar em harmonia com as forças primordiais da natureza yin e yang. O princípio básico da acupuntura sustenta que o equilíbrio é mantido no corpo humano por meio de fluxo de uma energia suave denominada gi, e do sangue, designado xue. O equilíbrio segundo essa ciência pode ser entendido como saúde e o desequilíbrio, como doença. Seguindo este princípio, qualquer tipo de disfunção ou patologia, como por exemplo, a ansiedade, podem ser tratados por intermédio da acupuntura, sendo que uma das formas de a eliminar é a inserção de agulhas em pontos específicos do corpo, para restabelecer o equilíbrio e o fluxo suave. Este artigo apresenta como foco de interesse e objetiva avaliar as evidencias sobre os efeitos da acupuntura no tratamento da ansiedade. Com isso, pretende-se identificar as melhores evidencias das publicações cientificas, para melhor entendimento da proposta apresentada na pesquisa.

\section{REVISÃO TEÓRICA}

A ansiedade, de acordo com a literatura é um sentimento vago e desagradável acompanhada pelo medo, tensão e desconforto derivado de antecipação de perigo de algo desconhecido ou estranho ao indivíduo. Considerada como uma emoção normal, comum do homem, é vista como um mecanismo de defesa de caráter adaptativo, que possui o papel de mediar à interação do homem com o meio ambiente. Os autores Vasconcelos et al. (2008) enfatizam que a ansiedade é um sinal de alerta, porque adverte o homem sobre os perigos eminentes e o impulsiona a tomar medidas para enfrentá-la.

Segundo Silva (2010), não podemos considerar a ansiedade como um fenômeno patológico, sim uma função natural do organismo, permitindo ao homem estar preparado ou se preparar para responder, da melhor forma possível a uma situação desconhecida, ou a uma já conhecida e interpretada como potencialmente perigosa. Por outro lado, podemos elucidar que quando a ansiedade atinge graus elevados e contínuos, ela se torna prejudicial ao organismo, ou seja, ele continua em constante sinal de alerta, 
configurando, assim, segundo a literatura as patologias de transtornos de ansiedade (ARAÚJO, 2011).

Em conformidade com o estudo os autores Zamignani et al. (2005), acordam quando dizem que a ansiedade pode ser definida enquanto quadro clínico, quando interfere o comprometimento ocupacional do homem, impedindo os afazeres diários do mesmo, como: as atividades profissionais, sociais e acadêmicas. Para Souza (2012), os transtornos da ansiedade na atualidade são muitos frequentes, isto se caracteriza pelos estados subjetivos desagradáveis de inquietação, tensão e apreensão, com uma certa tendência a cronicidade, trazendo sérios transtornos ao cotidiano dos indivíduos, e isto tem aumentado estudos entre vários grupos de pessoas. Estes estudos tornam-se importantes pois esta enfermidade possui altos índices de prevalência na população, refletindo sobremaneira na saúde pública, aos altos custos sociais e individuais, com uma demanda elevada de assistência.

Podemos referenciar o transtorno de ansiedade em várias situações, como: ansiedade generalizada, síndrome do pânico, estresse pós-traumático, fobias, transtorno obsessivo-compulsivo, doenças da artéria coronária, disfunções gastrointestinais e asma (PILKINGTON, 2010). Segundo a OMS - Organização Mundial da Saúde, no Brasil cerca de $23,9 \%$ da população sofrem algum transtorno de ansiedade, sendo que o país tem a maior taxa no mundo, e é o quinto em casos de depressão. Cerca de 264 milhões de pessoas sofrem com transtorno de ansiedade, uma média de 3,6\% da população global. Os índices superam as taxas de outras regiões do país. No Paraguai, por exemplo a taxa é de $7,6 \%$, contra $6,5 \%$ no Chile e $6,4 \%$ no Uruguai. Em números absolutos, o Sudeste Asiático é a região que mais registra casos de transtornos de ansiedade: 60 milhões, $23 \%$ do total mundial. No segundo lugar vêm as Américas, com 57,2 milhões e $21 \%$ do total.

A ansiedade torna-se um grande problema quando ela passa a ser rotineira, a partir daí que devemos ficar alertas. Os distúrbios da ansiedade são encadeados por uma desordem do sistema nervoso simpático, que libera, na circulação, grandes quantidades inadequadas de hormônios que desencadeiam o estresse.

A ansiedade é mais comum em mulheres do que em homens, viciados em jogos ou internet, grávidas, pessoas mais jovens. Existem fatores que podem influenciar uma pessoa a desenvolver o estado de ansiedade: a genética, histórico de transtorno de ansiedade na família; traumas passados: como perda de um ente e acidentes marcantes; problemas de infância; Doenças físicas; problemas cardíacos como as arritmias, doenças hormonais como hipertireoidismo e hiperadrenocorticismo, problemas respiratórios como doenças pulmonares obstrutiva crônica. Dores físicas crônicas, abuso de drogas, álcool ou medicação, como benzodiazepínicos.

As pesquisas relativas a ansiedade são de fundamental importância, pois esta enfermidade possui altos índices de prevalência na população, seja, em qualquer período da vida, ela reflete na saúde pública devido aos altos custos sociais e individuais, o que ocasiona uma demanda elevada de assistência (SNIEZEK et al., 2013; SILVA, 2010; PRADO et al., 2012). Esta assistência é baseada a visão biológica do processodoença, atingindo todas as áreas da saúde que predomina a hegemonia de médicos sobre os demais profissionais, como também as tecnologias de altas densidade em nível secundário e terciário (HADDAD et al., 2012).

Dentre os tratamentos dos transtornos de ansiedade, os farmacológicos e psicoterápicos são mais 
utilizados, destaque para o benzodiazepínico, medicamento mais prescritos no mundo, utilizados como ansiolíticos e hipnóticos. Estes fármacos podem causar dependência física, química e psicológica, se o uso for abusivo ou prolongado, é necessário haver precauções (MARCHI et al., 2013). Paraguaná et al. (2009), diz que há várias discussões acerca da prática assistencial, com a finalidade de mudar o foco do cuidado curativista e farmacoterapêutico, os autores citam as terapias complementares, como mais uma forma de promover a integridade do cuidado.

No ano de 2016, no Brasil, as terapias complementares foram priorizadas como condutas terapêuticas no Sistema Único de Saúde - SUS. Isto foi possível por meio da homologação da Política Nacional de Práticas Integrativas e Complementares o - PNPIC. A recomendação do Ministério da Saúde e que essas práticas, sejam usadas no âmbito da Atenção Primária à Saúde, como uma nova estratégia para a promoção, manutenção e a recuperação da saúde (BRASIL, 2006).

Dentre as terapias complementares, destaca-se a acupuntura, uma técnica milenar da Medicina Tradicional Chinesa, sua finalidade é o diagnóstico de doenças e o tratamento é realizado a partir do estímulo da força de auto cura do corpo. Foi por meio dos jesuítas que a acupuntura surgiu na China e se expandiu para os países da Europa, no século XVl; é considerada como o mais antigo método de tratamento do mundo, utilizado há mais de 300 anos. É uma técnica com princípios de tratamento que visa produzir efeitos terapêuticos e analgésicos (FREIRE, 2008; MORI, 2006).

A palavra acupuntura tem derivação dYAos radicais latinos acus (agulha) e puntura (picada), que resulta em puncionar com agulha. $\mathrm{O}$ tratamento por meio da acupuntura consiste na aplicação de agulhas, como forma de tratamento das patologias, estas aplicações de agulhas, promovem estímulos sobre a pele, em pontos estratégicos chamados acupontos. Os acupontos têm relação com as terminações nervosas sensoriais em determinadas regiões da pele, o que estabelece uma relação íntima com nervos, vasos sanguíneos, tendões, periósteos e cápsulas articulares (MORI, 2006, CLAUDINO, 2009).

Na teoria da acupuntura, todas as estruturas do organismo se encontram originalmente em equilíbrio pela atuação das energias Yin e Yang (Lei universal do mundo material: Todo objeto ou fenômeno no universo consiste de dois aspectos opostos Yin e Yang, que estão em conflito e interdependência. O Yang cria, o Yin retém e acrescenta.

Os pontos de acupuntura estão localizados ao longo de canais específicos cuja puntura nesses locais ajuda e restaura o fluxo de energia do corpo. Ou seja, ele se baseia no controle do fluxo de energia por meio de canais que estabelecem o equilíbrio do corpo (Ying e Yang), em que a doença é um reflexo da perda de harmonia, sendo assim utilizada no tratamento de desordens clínicas na China por mais de cinco mil anos (BELMIRO et al., 2013).

Os pontos de acupuntura são a expressão dos Zang Fu ao nível mais externo, e por meio dessa relação, é possível atuar no exterior para tratar e fortalecer os Zang Fu, situados no interior. Funcionam como meio de comunicação entre o exterior e o interior do corpo, com isso esses pontos estão sujeitos diretamente a influências das energias transmitindo-as para os canais de energia principais conduzindo aos Zang Fu (órgãos e vísceras) e destes para os tecidos (YAMAMURA, 2001). Os estímulos provocados pela agulha em 
diferentes receptores nervosos levam a múltiplos efeitos, sendo liberados vários neurotransmissores, resultando em diferentes interpretações cerebrais distintas e diferentes respostas. Com origem há mais de 4.000 anos a acupuntura passou a ser utilizada no Japão e na Coreia do Norte e do Sul, expandindo também para a Ásia. Desde a década de 70 essa terapia vem sendo questionada a sua eficácia (HADDAD et al., 2012; KUREBAYASHI et al., 2009).

Segundo Kaplan (1997), a ciência ocidental, ainda não há uma resposta imediata das causas da ansiedade, mais há estudos de base biológicos avançados, para melhor explicação sobre os transtornos de ansiedade. As pessoas que apresentam a ansiedade apresentam geralmente um sofrimento e sintoma específicos nos campos somáticos, motor, no humor e na cognição, é um sentimento que se apresenta, como se o indivíduo esteja sendo condenado constantemente. Desse modo ele apresenta sensações de algo terrível vai acontecer, o indivíduo apresenta sensações de tensão, medo, irritabilidade e depressão. Já os sintomas cognitivos, apresentam como à apreensão e/ou preocupação com uma possível condenação ou desastre que pode vir a ocorrer e que o indivíduo antecipa.

Segundo a literatura a acupuntura melhora de maneira significativa os transtornos de ansiedade, auxiliando ao paciente maior domínio de seus sentimentos. O tratamento pode ser associado à auriculoterapia, fitoterapia, homeopatia, terapia com florais entre outras técnicas. Há mais de 4.000 anos a auriculoterapia teve sua origem na China, ela consiste na aplicação com esferas, de sementes ou agulhas, no pavilhão auricular, que é encontrado em cada ponto da orelha um órgão correspondente. Esta técnica foi descoberta na escola francesa, a área auricular é um reflexo do Sistema Nervoso Central (SNC), nestes sistemas as patologias e os distúrbios aparecem em uma área determinada, e quando é estimulado estes pontos, no cérebro é liberado os neurotransmissores e hormônios que irão atuar sobre o distúrbio ou a patologia que vai ser tratado (MOURA, 2015).

Ross (2003), colabora com o estudo quando diz que na Medicina Tradicional Chinesa MTC, a ansiedade está ligada com o coração por excesso, deficiência e estagnação com o Rim. Com outras emoções também do coração como agitação, pânico, histeria ou insônia, palpitações, hipertensão, dor na região cardíaca entre outras. $O$ autor é enfático quando cita que os distúrbios de ansiedade estão relacionados ao desequilíbrio entre os sistemas do coração e do rim, podendo ela está combinada com sobressaltos e receio, de que algo terrível irá acontecer, como sinais físicos, tremor, frequência urinária ou intestinos soltos.

O autor cita que, a ansiedade é o resultado de um distúrbio do shen, que é um sintoma que indica que o espírito não está conseguindo mover-se adequadamente pelo corpo. Ele cita que na perspectiva chinesa, existem três tipos diferentes de ansiedade: a ansiedade por excesso; por estagnação e por deficiência. Ross (2003), diz quando o coração está agitado em excesso e o rim debilitado, e a água do rim não consegue acalmar o fogo do coração, e o shen ficou agitado, surge os sintomas característico da ansiedade. Para reverter o quadro é necessário o tratamento pela medicina tradicional chinesa, por meio da inserção de agulhas em pontos específicos do corpo que, combinados, apresentam essa finalidade, ou seja, pela acupuntura.

De acordo com os autores Campiglia (2004) e Vectore (2005), o tratamento realizado por meio da 
acupuntura não proporciona curas milagrosas ou o fim total das patologias dos pacientes. 0 restabelecimento da saúde se dá de modo gradual e está diretamente relacionado a condições externas (ambientais, climáticas, sociais e históricas) e internas (alimentação, estados emocionais, espiritualidade), com as quais o sujeito se relaciona. O tratamento é processual, e a paciente deverá fazer as sessões cotidianamente, mesmo a se mesma relatar que não sente mais os sintomas de ansiedade, faz necessário que esteja em tratamento, ainda mais se a mesma apresentar alterações nos pulsos.

\section{METODOLOGIA}

Este estudo foi realizado por meio de pesquisa bibliográfica. Para Prestes (2008), "a pesquisa bibliográfica busca adquirir conhecimentos a partir de informações provenientes de materiais gráficos ou de outras fontes". A pesquisa bibliográfica parte do problema relatado a partir das referências teóricas científicas publicadas em documentos, nesse método de pesquisa se busca estudar e analisar teorias científicas de um determinado assunto, tornando-se instrumento essencial para estudos monográficos.

Esta pesquisa deve ser exaustiva sobre o tema em questão, leituras diárias, seleção de conteúdo, mapeamento dos mesmos, pois uma pesquisa bibliográfica tem muitos objetivos como: fazer um histórico sobre o tema atualizar-se sobre o mesmo, encontrar respostas ao problema formulado, levantar contradições sobre o tema, e evitar repetições em trabalhos acadêmicos já realizados.

Para a elaboração desta revisão foram realizadas buscas nas bibliotecas virtuais Lilacs, Bireme, Scielo, Pubmed e Medline a fim de se verificar artigos disponíveis na língua portuguesa e inglesa. A seleção dos artigos foi feita em conformidade com assunto proposto, critérios de inclusão foi sendo destacados, por meio dos escritores chaves 'Ansiedade' 'Acupuntura' e 'Medicina Chinesa', outro foi literaturas inclusas dos últimos vinte anos 1997 até 2018.

\section{RESULTADOS E DISCUSSÃO}

Para elaboração desta pesquisa foram verificados artigos, compreendendo os anos de 1997 a 2018, devido a estes serem os que abordaram satisfatoriamente o tema em estudo. Colaborando com o estudo de acordo com Organização Mundial da Saúde, indica a acupuntura para o tratamento da ansiedade com eficácia superior à medicação convencional. Além de ser segura para o tratamento é de fácil aplicabilidade, não é tóxica, e não leva a dependência. Não possuindo contraindicações e de procedimento simples e de baixo custo.

Errington-Evans (2015), ressalta que deva haver uma definição do número de sessões e tempo de terapia de novas pesquisas, segundo o mesmo, esta preocupação vem ao encontro de melhorar a padronização e fornecer o tratamento ideal. Por meio da ariculoterapia, Moura et al. (2015), realizou uma pesquisa, utilizando os pontos Shernmem, relaxamento e pulmão, onde obteve um resultado satisfatório para a redução da ansiedade. Diante disso, evidências científicas neste estudo mostram efeitos positivos e estatisticamente significativos do uso da acupuntura para tratamento de indivíduos com ansiedade. 


\section{CONCLUSÕES}

Após as leituras do tema proposto, os resultados da pesquisa mostram que a acupuntura auxilia no tratamento da ansiedade, na atualidade várias pessoas buscam uma terapia alternativa e natural para aliviar a ansiedade. A acupuntura promove o equilíbrio físico, biológico e mental, como também aumenta a produção de endorfina e serotonina, que são responsáveis pela sensação de bem-estar, sem agredir o corpo e a mente, como os remédios químicos.

Na literatura existem diversos estudos científicos comprobatórios de que a acupuntura é eficaz ao tratamento da ansiedade, podendo ser facilmente associada a outros tipos de tratamentos como medicamentoso e a psicoterapia. O tratamento da ansiedade por meio da acupuntura proporciona ao paciente uma sensação de leveza, que é induzida pelos estímulos dos pontos de acupuntura.

O tratamento da ansiedade por meio da acupuntura não é uma forma milagrosa, é preciso que gradualmente o paciente deixe de fazer a aplicação rotineiramente, mais, não deve deixar de lado o tratamento, sendo necessário que passe por uma sessão uma vez ou outra. A Medicina Tradicional Chinesa é mais um somatório no tratamento das doenças do corpo e da alma. É preciso que haja mais estudos sobre o assunto, para que novas pesquisas sejam um somatório na comprovação de que a acupuntura é eficaz no tratamento.

\section{REFERÊNCIAS}

ARAÚJO, N. G.. Fobia específica: passo a passo de uma intervenção bem-sucedida. Revista Brasileira de Terapia Cognitiva, v.7, n.2, 2011.

BELMIRO, H.; OLIVEIRA, D. V.; CAMILLOTI, C. M.. Efeitos da Acupuntura no Desempenho Motor de Atletas. Revista da Faculdade de Educação Física da UNICAMP, Campinas, v.11, n.3, p.176-191, 2013.

BRASIL. Ministério da Saúde. Portaria n. 971, de 4 de maio de 2006. Aprova a Política Nacional de Práticas Integrativas e Complementares (PNPIC) no Sistema Único de Saúde. Brasília: DOU, 2006.

CAMPIGLIA, H.. Psique e medicina tradicional chinesa. São Paulo: Roca, 2004.

CLAUDINO, A.. Apostila de teorias básicas da medicina tradicional chinesa acupuntura bioenergética. Santa Clara: Escola Catarinense de Terapias Naturais, 2009.

ERRINGTON-EVANS, N.. Acupuncture for Anxiety. CNS Neurosci Ther, 2012.

FREIRE, M.. Acupuntura Tradicional Chinesa. São Paulo: Livro Pronto, 2008.

HADDAD, M. L.; MEDEIROS, M.; MARCON, S. S.. Sleep quality of obese workers of a teaching hospital: acupuncture as a complementary therapy. Rev. Esc. Enferm. USP, v.46, n.1, 2012.

KAPLAN, H. I.; SADOCK, B. J.; GREBB, J. A.. Compendio de Psiquiatria: Ciência do Comportamento e Psiquiatria Clinica.
7 ed. Porto Alegre, 1997.

KUREBAYASHI, L. F. S.; FREITAS, G. F.; OGUISSO, T.. Nurses perception about diseases that are treated by acupuncture. Rev. Esc. Enferm. USP, v.43, n.4, 2009.

MARCHI, K. C.; BÁRBARO, A. M.; MIASSO, A. I.; TIRAPELLI, C. R.. Anxiety and the consumption of anxiolytics among nursing students of a public university. Rev. Eletrônica Enferm., v.15, n.3, p.729-37, 2013.

MORI, H.. Introdução a Acupuntura. São Paulo: Ícone, 2006.

MOURA, C. C.; CARVALHO, C. C.; SILVA, A. M.; IUNES, D. H.; CARVALHO, E. C.; LOPES, E. C.. Efeito da Auriculoterapia na Ansiedade. Alfenas, 2015

PARAGUANÁ, T. T. B.; BEZERRA, A. L. Q.; SOUZA, M. A.; SIQUEIRA, K. M.. As práticas integrativas na Estratégia Saúde da Família: visão dos agentes comunitários de saúde. Rev. Enferm. UERJ, v.17, n.1, p.75-80, 2009.

PRADO, J. M.; KUREBAYASHI, L. F. S.; SILVA, M. J. P.. Auriculotherapy effectiveness in the reduction of anxiety in nursing students. Rev. Esc. Enferm. USP, v.46, n.5, p.12001206, 2012.

PILKINGTON, K.. Anxiety, depression and acupuncture: a review of the clinical research. Auton Neurosci, v.157, n.1-2, p.91-5, 2010.

PRESTES, M. L. M.. A pesquisa e a construção do conhecimento científico: do planejamento aos textos, da escola à academia. 3 ed. São Paulo: Rêspel, 2008. 
ROSS, J.. Combinações dos pontos de acupuntura: a chave para o êxito clínico. São Paulo: Roca, 2003.

SILVA, A. L. P.. O Tratamento da Ansiedade por intermédio da Acupuntura: Um Estudo de Caso. Psicologia Ciência e Profissão, 2010.

SNIEZEK, D. P.; SIDDIQUI, I. J.. Acupuncture for treating anxiety and depression in women: a clinical systematic review. Med Acupunct, v.25, n.3, 2013. DOI:

https://doi.org/10.1089/acu.2012.0900

SOUSA, L. G.. O auxílio da acupuntura no tratamento dos sintomas da ansiedade e depressão. Faculdade FASAM, 2012.
VASCONCELOS, A. S.; COSTA, C.; BARBOSA, L. N. F.. Do transtorno de ansiedade ao Câncer. Revista SBPH, v.11, n.2, 2008.

ZAMIGNANI, D. R., BONACO, R. A.. Um panorama analíticocomportamental sobre os transtornos de ansiedade. Revista Brasileira de Terapia Comportamental, v.7, n.1, 2005.

VECTORE, C.. Psicologia e acupuntura: primeiras aproximações. Psicologia: Ciência e Profissão, v.25, n.2, p.266-285, 2005.

YAMAMURA, Y.. Acupuntura Tradicional: A Arte de Inserir. 2 ed. São Paulo: Roca, 2001.

A CBPC - Companhia Brasileira de Produção Científica (CNPJ: 11.221.422/0001-03) detém os direitos materiais desta publicação. Os direitos referem-se à publicação do trabalho em qualquer parte do mundo, incluindo os direitos às renovações, expansões e disseminações da contribuição, bem como outros direitos subsidiários. Todos os trabalhos publicados eletronicamente poderão posteriormente ser publicados em coletâneas impressas sob coordenação da Sustenere Publishing, da Companhia Brasileira de Produção Científica e seus parceiros autorizados. Os (as) autores (as) preservam os direitos autorais, mas não têm permissão para a publicação da contribuição em outro meio, impresso ou digital, em português ou em tradução. 